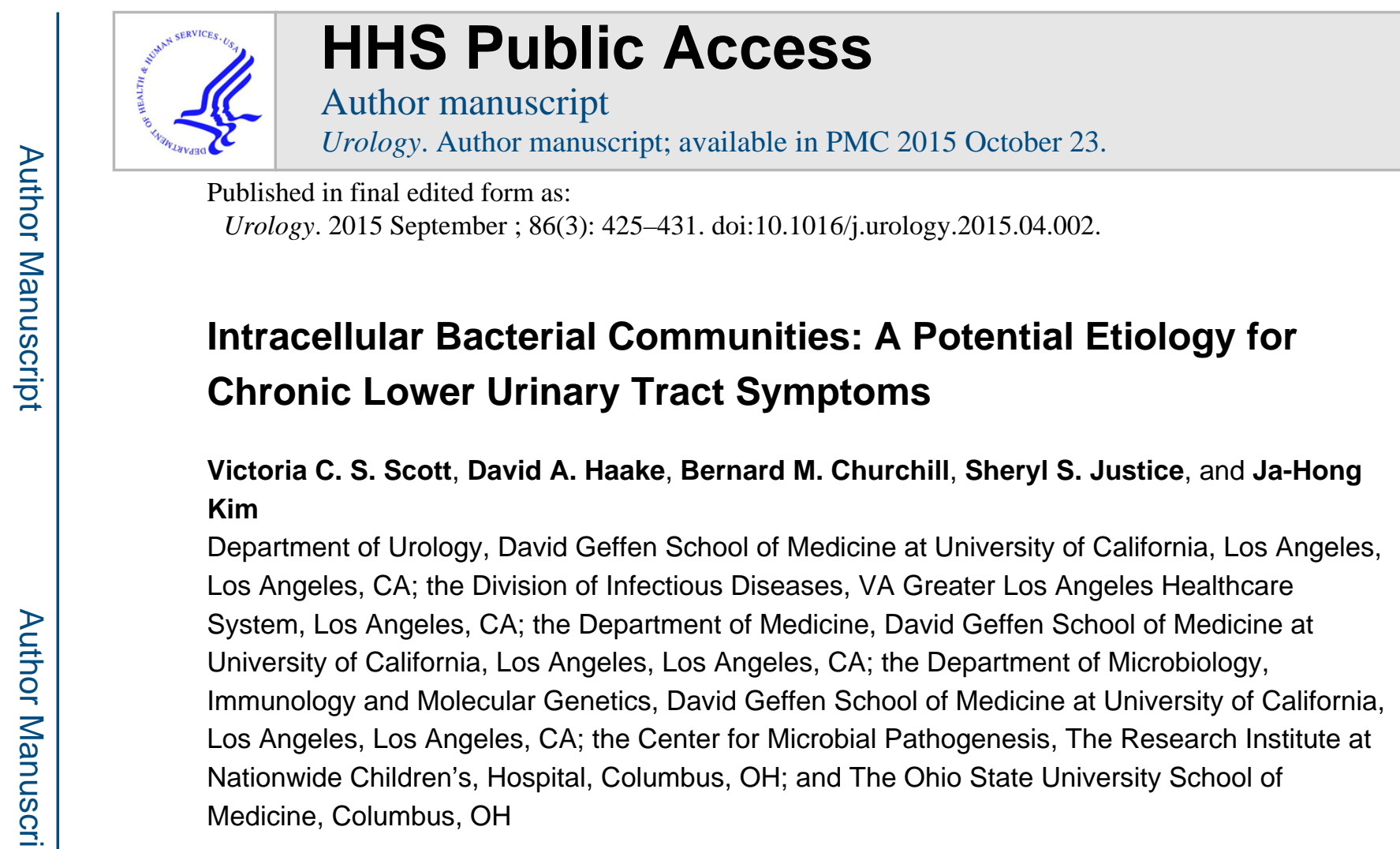

\begin{abstract}
Patients with persistent lower urinary tract symptoms and negative urine cultures are often difficult to treat. Infection may go undetected in these patients because the concentrations of bacteria in their urine are beneath the threshold of standard urine culture techniques. Empiric treatment may result in temporary relief, followed by recurrent symptoms. Occult and recurrent urinary tract infection may be due to both invasion of the bladder wall by uropathogenic Escherichia coli and the formation of biofilm-like intracellular bacterial communities. This review examines emerging evidence for a role of intracellular bacterial communities in human infection.
\end{abstract}

Lower urinary tract symptoms (LUTS), such as frequency, urgency, and dysuria, are extremely prevalent among adults worldwide. In an international study of 30,000 participants aged 40 to 99 years, $72.3 \%$ of men and $76.3 \%$ of women reported at least one LUTS "sometimes," and $47.9 \%$ of men and $52.5 \%$ of women reported at least one LUTS "often". ${ }^{1}$ Patients with isolated or repeated episodes of LUTS associated with positive urine cultures are often effectively treated with short courses of antibiotics. Yet, no etiology for LUTS is found in many patients with negative results using standard urine culture techniques, who also lack a functional or anatomic abnormality of the urinary tract. Patients with urgency as their primary complaint are typically thought to have overactive bladder $(\mathrm{OAB})$, and patients with pain, pressure, or discomfort are diagnosed with interstitial cystitis/bladder pain syndrome (IC/BPS). OAB and IC/BPS are diagnoses of exclusion for

Address correspondence to: Victoria C. S. Scott, M.D., Department of Urology, David Geffen School of Medicine at UCLA, 10833 Le Conte Ave, Box 951738, Los Angeles, CA 90095. vscott@mednet.ucla.edu.

Financial Disclosure: David Haake filed invention disclosures with the University of California, Los Angeles (UCLA) and the Veteran Affairs Association, and is the cofounder of Qvella, a clinical microbiology diagnostics company. Bernard Churchill filed invention disclosures with UCLA and the Veteran Affairs Association, is the co-founder of Qvella, a clinical microbiology diagnostics company, and is a consultant and stockholder of NovaBay Pharmaceuticals Inc. The remaining authors declare that they have no relevant financial interests. 
which there is frequently no clear etiology. ${ }^{2}$ Treatment of such patients is targeted at symptom management, with little hope of a definitive cure.

There is great interest in the potential role of urothelial cell infection in patients with LUTS who have $\leq 10^{5}$ colony-forming units (CFU) of bacteria per milliliter of urine ("low-count" bacteriuria) or even negative urine cultures. Urinary tract infection in some patients may not be limited to a simple luminal infection that is reliably eradicated with a short course of antibiotics. A growing body of evidence indicates that the pathogenesis of infections caused by uropathogenic Escherichia coli (UPEC) may be far more complex and include invasion of urothelial cells lining the urinary bladder with formation of intracellular bacterial communities (IBCs). ${ }^{3-6}$ In this biofilm state, IBCs may go undetected by standard urine cultures, evade host defense mechanisms, and persist despite antibiotic therapy. In this report, we explore the potential role of occult infection in OAB and IC/BPS, review the evidence for IBC formation in human bladder infections, and discuss the possible role of IBCs in recurrent UTIs.

\section{“CULTURE-NEGATIVE” LUTS}

Patients with symptoms of urinary urgency, frequency, dysuria, or bladder pain who have normal functional and anatomic studies and negative urine cultures are frequently given the diagnosis of OAB or IC/BPS. OAB and IC/BPS are both symptom complexes, not diseases, which rely on negative urine cultures for diagnosis. A negative urine culture using the standard definition of $₫ 0^{5} \mathrm{CFU} / \mathrm{mL}$ result does not exclude "occult" UTI involving lowcount bacteruria or "latent" infection involving quiescent reservoirs in the urothelium.

The International Continence Society defines OAB as urgency, with or without urgency incontinence, usually with frequency and nocturia, in the absence of infection or other pathology. ${ }^{2}$ Urgency, along with at least 1 other symptom, must be present for a diagnosis of $\mathrm{OAB}$. There are 2 primary hypotheses for the etiology of $\mathrm{OAB}$. The neurogenic hypothesis states that detrusor overactivity arises from generalized, nerve-mediated excitation of the detrusor muscle, whereas the myogenic hypothesis suggests that overactive detrusor contractions result from a combination of increased likelihood of spontaneous excitation within bladder smooth muscle and propagation of this activity to an excessive proportion of the bladder wall. ${ }^{7,8}$

IC/BPS is less common but remains a debilitating condition for many women and some men. ${ }^{9}$ Although IC/BPS and OAB share the symptoms of urgency, frequency, and nocturia, pain is the hallmark symptom for IC/BPS. It can also be associated with chronic dysuria. IC/BPS encompasses a major portion of the "painful bladder" disease complex, which includes any or all of bladder, urethral, or pelvic pain, LUTS, and negative urine cultures. Multiple theories to explain the pathogenesis of IC/BPS have been proposed, including a "leaky epithelium," neurogenic inflammation, hypersensitivity response with mast cell activation, or some combination of these, leading to chronic bladder pain and voiding dysfunction. ${ }^{10}$

A number of studies have attempted to demonstrate an infectious etiology for OAB and IC/ BPS. ${ }^{11-14}$ Infection may be the initial insult to the urothelium that ultimately results in 
development of OAB or IC/BPS. UTI is the most common initial diagnosis in patients who are eventually diagnosed with IC/BPS, ranging from $42 \%-60 \%$ of patients. Patients with IC/BPS are twice as likely to report a history of UTI before the onset of IC/BPS symptoms compared with patients who do not have IC/BPS and 10- to 12-times as likely to have a history of childhood urinary tract disorders. ${ }^{14} \mathrm{OAB}$ and IC/BPS may be caused by chronic infection or may be a result of an initial infection causing persistent inflammation of the bladder after the infection has been eradicated.

There has been interest in examining the response of OAB or IC/BPS patients to empiric antibiotics. In the most successful study of antibiotic treatment of LUTS, Durier ${ }^{15}$ reported cure of 27 of 27 patients with IC with use of up to 5 sequential antibiotics. Warren et al ${ }^{16}$ performed a prospective, double-blind, placebo-controlled randomized trial of 50 women diagnosed with IC who were given placebo or an intensive antibiotic regimen for 18 weeks. Although the difference did not reach statistical significance, $88 \%$ of patients in the antibiotic group reported overall improvement or improvement in pain and urgency compared with 44\% in the placebo group. These results suggest that the LUTS in a subset of IC/BPS patients has an infectious etiology despite negative urine cultures. An alternative interpretation is that relief of symptoms results from the placebo effect or the antiinflammatory activity of some antibiotics, such as tetracyclines.

Infectious causes of urethritis may also play a role in some IC/BPS patients with negative urine cultures. Burkhard et al ${ }^{17}$ treated 103 women, aged 21 to 84, with a median duration of LUTS for 60 months, with doxycycline, $100 \mathrm{mg}$ twice daily for 2 weeks, followed by 100 $\mathrm{mg}$ once daily for 2 weeks. Patients were also given vaginal tablets (hexetidine or ciclopiroxolamine) once daily for 10 days. Patients' sexual partners were treated with the same doxycycline regimen. After 4 weeks, $70 \%$ of women reported subjective cure or improvement in symptoms. In another study of 48 women referred for chronic voiding symptoms and possible IC with negative standard urine cultures, $48 \%$ had cultures positive for Ureaplasma urealyticum or Mycoplasma hominis. ${ }^{18}$ After treatment, $91 \%$ of the patients with positive cultures had statistically significant improvement in their symptoms.

\section{MICROBIOLOGIC DIAGNOSIS OF UTI}

Acute UTI often presents with bacteria in the urine at $\geq 10^{5} \mathrm{CFU} / \mathrm{mL}$, which is the standard threshold for a positive urine culture. This density cutoff was determined in studies of women with acute pyelonephritis vs asymptomatic bacteriuria in the 1950s. Lower densities of bacteria were more likely to be associated with contamination from nonurinary sources. ${ }^{19}$ The threshold of $\geq 10^{5} \mathrm{CFU} / \mathrm{mL}$ shows high specificity for a UTI but a sensitivity of only $\sim 50 \%$. Lowering the threshold to $\geq 10^{3}$ in women with symptoms of cystitis increases sensitivity considerably, with minimal reductions in specificity. ${ }^{20,21}$ In fact, the guidelines of the European Association of Urology and the American Academy of Family Physicians recommend $10^{3}$ or $10^{2} \mathrm{CFU} / \mathrm{mL}$, respectively, as the standard for diagnosing lower UTI in symptomatic patients. ${ }^{22,23}$

Multiple studies have shown that a substantial number of women with symptoms of acute cystitis and pyuria have $<10^{5} \mathrm{CFU} / \mathrm{mL}$ on urine culture. ${ }^{24,25}$ One study of 202 
premenopausal, nonpregnant women who presented with at least 2 symptoms of acute cystitis compared colony formation between a clean-catch urine specimen and a catheterized urine specimen. ${ }^{25} \mathrm{E}$ coli growth was found in the catheterized specimen of $60 \%$ of patients, with $40 \%$ of these women found to have $<10^{5} \mathrm{CFU} / \mathrm{mL}$ in the voided specimen. The presence of $\mathrm{E}$ coli in midstream urine with $10^{2} \mathrm{CFU} / \mathrm{mL}$ had a $93 \%$ positive predictive value for bladder bacteriuria. An earlier study performed in a similar fashion reported the best diagnostic criteria was $\geq 10^{2} \mathrm{CFU} / \mathrm{mL}$, which allowed for a sensitivity of $95 \%$ and a specificity of $85 \%$ for the diagnosis of bacteriuria in symptomatic women. ${ }^{20}$

Interestingly, all other diagnostic tests of urine constituent levels normalized with creatinine to account for dilution of the urine. However, laboratory diagnostics for bacterial burden only report the CFU/mL of urine. Taking account of urinary creatinine levels is particularly relevant considering that most women increase water intake during UTI to aid in clearance. The intracellular paradigm for IBC formation during UTI further complicates the interpretation of the culture-based diagnosis of UTI.

First, bacterial density in the urine may not correlate with the numbers of bacteria in the bladder wall. Significant numbers of bacteria can remain within the tissue even in the absence of bacteriuria.

Second, a single exfoliated urothelial cell can contain $\sim 10^{5} \mathrm{CFU}$ of bacteria but would only be enumerated as a single colony by traditional culture-based methods unless the sample were properly homogenized to release the bacteria within the IBC.

Lastly, culture-based methods underestimate the bacterial burden when filamentous populations are present. A single filament of $70 \mu \mathrm{m}$ has the biomass and the genetic content of 35 bacteria but would translate to only $1 \mathrm{CFU}$. These confounding issues underscore the need for new diagnostic approaches.

Innovative methods of identification of uropathogens have emerged, particularly for female patients with LUTS. ${ }^{26-28}$ Through expanded quantitative urine culturing and 16S ribosomal ribonucleic acid (RNA) sequencing, studies have characterized the "female urinary microbiome" and demonstrated that the urine of most patients is not sterile. OAB patients and controls underwent transurethral catheterization to avoid contamination of the urine with urethral or periurethral flora. Expanded quantitative culture techniques showed that $80 \%$ of the urine samples from symptomatic and asymptomatic patients with negative results using standard culture techniques grew bacterial species, including Lactobacillus (15\%), Corynebacterium (14.2\%), Streptococcus (11.9\%), Actinomyces (6.9\%), and Staphylococcus $(6.9 \%) .{ }^{26}$ There is not yet substantial evidence for a significant difference in the presence of specific bacterial species or of a certain quantity of bacteria between healthy patients and those with LUTS. However, there is evidence to suggest that these differences in the urinary microbiome may playa role in female urinary tract health. When the urinary microbiome in patients with and without urge urinary incontinence were compared using sequencing of $16 \mathrm{~S}$ ribosomal RNA, women with urge-urinary incontinence had statistically significant increased numbers of Gardnerella and decreased Lactobacillus compared with asymptomatic women. ${ }^{27}$ 


\section{THE ROLE OF UROTHELIAL INVASION IN UTI}

Recent research in animal models has shown that UPEC, which accounts for 70\%-90\% of human UTIs, is capable of forming biofilm-like communities within superficial bladder epithelial cells in the setting of cystitis (Fig. 1) ${ }^{5,29}$ These IBCs are difficult to detect in urine specimens, can remain quiescent for extended periods despite antibacterial therapy, and can re-emerge to cause recurrent infection. ${ }^{30,31}$ In this scenario, the bladder wall itself may serve as a reservoir for infection of the urinary tract lumen. There is evidence that other organisms, such as Enterococcus faecalis and Klebsiella pneumonia, can also form IBCs in experimental models of UTI..$^{32,33}$

Adherence to the host urothelium is a crucial step in the pathogenesis of UTI. ${ }^{5}$ UPEC binds to mannose-containing glycoprotein receptors on superficial bladder urothelial cells via FimH, an adhesin located at the distal end of type 1 pili. Adherence to the urothelium protects the UPEC from being washed out of the urinary tract by countering urinary shear forces during bladder emptying. Once bound, the epithelial cells internalize UPEC with their own endocytotic pathway. This internalization allows the bacteria to enter the nutrient-rich intracellular environment where they are protected from antibiotics, neutrophil influx, and shear stress. ${ }^{33}$ Binding and internalization of UPEC activates host inflammatory and apoptotic cascades in the epithelium. Neutrophil influx into the bladder is mediated by the Toll-like receptor 4eCD14 pathway recognizing bacterial lipopolysaccharide.

Three distinct stages of intracellular infection have been characterized in the mouse model: early, middle, and late IBCs. Beginning at 6 hours after bladder inoculation, loose intracellular collections of bacteria, IBCs or bacterial "factories," have been found in the superficial cells of the bladder. ${ }^{34}$ These collections of bacteria underwent a developmental cascade within the cytoplasm of urothelial cells. At 24 hours after infection, intracellular communities with a biofilm-like appearance were noted, creating a visible bulge in the luminal surface of urothelial cells. These bulges were termed "pods." Later in the cascade, the IBCs were noted to break open, resulting in bacterial dispersal. ${ }^{3}$

\section{Early Stage: IBC Formation}

After invasion of the superficial umbrella cells of the bladder, bacteria replicate rapidly and form a loose collection. ${ }^{4}$ The rapid growth has been observed for up to 8 hours, with UPEC maintaining the rod shape ( $\_\mu \mathrm{m}$ ) that is typically observed when grown in laboratory conditions. The rapid growth, with doubling times of 30-35 minutes, enables a surge of infectious bacteria in the bladder, which enhances the opportunity for the UPEC to disseminate and persist.

\section{Middle Stage: IBC Maturation}

Between 6-8 hours after infection, the UPEC growth rate slows to a doubling time of 60 minutes. The rod shape also appears to shorten to $0.7 \mu \mathrm{m}$ and form closely packed, tight communities. ${ }^{4}$ These dense communities grow to occupy an entire superficial umbrella cell, and in most cases, result in the development of pods. The increasing organization at this 
stage of development allows the bacteria to develop biofilm-like traits, making them resistant to antibiotic therapies.

\section{Late Stage: Fluxing and Release}

In the late stage of IBC formation, 12 hours after infection, UPEC differentiates into the typical rod shape, with an average length of $2 \mu \mathrm{m} .{ }^{4}$ UPEC also develops the ability to dissociate from the urothelial cells and flux into the bladder lumen, causing bacteriuria and allowing for spread and invasion of other bladder cells. This initiates a second round of invasion and differentiation. The motility of the UPEC is enabled by flagella, as observed on videomicroscopy. ${ }^{4}$ This stage has also been characterized by filamentous bacterial forms. Filamentous bacteria continue to grow in length with reduced septation, reaching up to 70 $\mu \mathrm{m}$ in cell length. Whether this process occurs on the bladder surface as well as within the IBC is uncertain, but septation of filamentous bacteria on the bladder surface to allow release of rod-shaped daughters has been noted on videomicroscopy. These daughters have the potential to invade other superficial umbrella cells.

\section{A Quiescent Reservoir}

Analysis of superficial umbrella cells 36-48 hours after infection showed smaller-thannormal bacteria and the presence of intracellular rod-shaped bacteria in the early stage of IBC formation. ${ }^{4}$ At subsequent times points 4, 5, 6, and 12 days later, the bacteria had the same phenotype. No bacterial growth was detected in the urine at this time, suggesting the establishment of a latent infection.

\section{Exfoliation of Cells}

Bladder epithelial cells typically have a very slow turnover rate, often longer than 200 days. ${ }^{35}$ However, in the urine of patients with UTIs, the presence of numerous exfoliated bladder cells suggests that exfoliation and removal of infected cells from the bladder serves as a host defense mechanism. ${ }^{5}$ Bacteria are shed into the urine along with sloughed cells. Exfoliation occurs via apoptotic mechanisms. In an effort to regenerate the exfoliated epithelium, transitional cells also undergo differentiation and proliferation.

\section{EVIDENCE FOR UROTHELIAL INVASION IN HUMANS}

Although most of the research on IBCs has been performed in murine models, there is a growing body of evidence that IBCs are relevant to human UTIs. UPEC strains cultured from the urine of women with acute cystitis, recurrent cystitis, asymptomatic bacteriuria, and pyelonephritis were able to progress through the IBC cascade in mouse models. IBC formation was noted in 15 of the 18 UPEC isolates collected from women in that study. ${ }^{36}$ Interestingly, 2 of the 3 isolates that did not show IBC formation when inoculated alone formed IBCs when inoculated with an IBC-competent isolate.

Filamentous bacteria and urothelial cells containing IBCs have been observed in urine samples from patients with UTI caused by UPEC, $K$ pneumoniae, and E faecalis. ${ }^{37-41}$ In one study, clean-catch urine specimens from 80 healthy young women with acute uncomplicated cystitis and urine specimens from 20 asymptomatic women with a history of UTI were 
examined by light microscopy, immunofluorescence, and electron microscopy. ${ }^{37}$ Of the 65 patients with $E$ coli infections, $22 \%$ had evidence of IBCs and $45 \%$ showed filamentous bacteria, whereas IBCs or filamentous bacteria were not found in the 20 asymptomatic participants. None of the urine samples with gram-positive growth showed IBCs or filamentous bacteria.

Similar studies have subsequently confirmed the presence of IBCs in other populations of patients as well. One study that evaluated children with $E$ coli cystitis found intracellular bacterial in $36.8 \%$ of the patients. ${ }^{38}$ In renal transplant recipients screened for UTIs, IBCs were visualized in 23 of 53 patients (44\%), with only 1 patient deemed to have a UTI by standard bacterial culture. ${ }^{39}$ Another study looked at women with LUTS compared with asymptomatic controls and found $75 \%$ of the LUTS patients had evidence of IBCs compared with $17 \%$ of the controls. Although none of the symptomatic patients had growth of $>10^{5}$ CFU on standard urine culture, $42 \%$ showed some growth of $E$ coli or E faecalis, or both, with the addition of a few other species. ${ }^{41}$

Given the evidence that bacteria may invade the urothelium to form IBCs, which can go undetected on urine culture and evade antibiotic therapy, we propose that IBCs may have a role not only in the etiology of recurrent UTI but also of chronic LUTS experienced by some women who are given the diagnosis of OAB or IC/ BPS. Bacteria with the ability to invade the urothelium may cause chronic LUTS due to persistent occult infection or perhaps due to chronic irritation and inflammation of the bladder wall from the initial insult of infection and invasion.

\section{FUTURE DIRECTIONS}

More translational research is urgently needed to determine whether IBCs form in human urothelial cells and are a mechanism for recurrent UTI, and possibly for persistent LUTS with or without positive urine cultures. In the laboratory, the mechanism for IBC formation and cycle has been clearly elucidated in the murine model of UTI. There are evolving data suggesting that IBCs in the human bladder may be associated with recurrent UTI. A better understanding of IBCs and occult UTIs may introduce a new paradigm and lead to more successful diagnostic and treatment modalities for patients with recalcitrant symptoms.

In addition to the vaginal and fecal reservoirs, the bladder wall should also be considered as a reservoir for reinfection. Exfoliated pods and filamentous bacteria, stages of the IBC cycle, have been identified in the urine of patients with positive and negative urine cultures. ${ }^{37-41}$ UTIs have a strong propensity to recur, and numerous studies have shown that most recurrent UTIs are caused by the same bacterial strain as the initial infection. ${ }^{42-44}$ More specifically, the same bacterial strain that caused an initial UTI has been identified in the urine of women with recurrent infections up to 3 years later. ${ }^{44}$ This recurrence pattern does not rule out a fecal or vaginal source for reinfection in some patients. However, a chronic infection model with the bladder as the reservoir should be considered when local and oral antibiotic prophylaxis fails to resolve the cycle of reinfections. Interestingly, the application of daily topical antibiotics to the perineum did not prevent recurrent episodes of UTIs. ${ }^{45}$ 
Given that IBCs cycle through intracellular phases, some patients with chronic LUTS may have repeatedly negative urine cultures despite the presence of occult infection (Fig. 2). In the IBC cycle, only the intraluminal stages are likely to yield bacterial growth on a standard urine culture, and the intracellular stages are unlikely to do so. Distinguishing these 2 stages of the IBC cycle is important because a UTI that is in the intraluminal stage and detectable on urine culture is more likely susceptible to antibiotic treatment. Although the intraluminal bacteria in these patients are cleared with antibiotics, some may evade complete eradication because IBCs can persist within the protected environment of the bladder wall and are thereby capable of causing reinfection in the future. With this concept of infection, the bladder urothelium must be considered as a dynamic surface in which there is constant interplay between pathogens and host factors.

The possibility of bacteria remaining in quiescent reservoirs in the bladder wall that are not detected when urine is cultured, as well as the emerging data that urine is not actually sterile, make interpretation of the urine culture as the gold standard for ruling out a UTI more challenging. To improve diagnostic sensitivity in patients with persistent LUTS, the microbiologic threshold for clinically significant bacteriuria should be re-examined. There is evidence that the threshold of $10^{5} \mathrm{CFU} / \mathrm{mL}$ for a positive urine culture leads to the underdiagnosis of bacteriuria in symptomatic women..$^{20,21}$ For women with persistent LUTS and negative bacterial urine cultures, we suggest asking the clinical microbiology laboratory to report all bacterial growth, regardless of quantity. Urine may also be cultured in larger volumes to enable growth of bacteria present in very low quantity. Given the prevalence of antibiotic-resistant uropathogens, recovery of pathogens for antibiotic susceptibility testing is preferred over empiric therapy. In addition, urine studies should be performed to rule out infections with pathogens not detected on routine cultures, including Ureaplasma urealyticum and $U$ parvum, $M$ hominis and $M$ genitalium, Chlamydia trachomatis, Neisseria gonorrhoeae, and viruses.

Despite the emerging data suggesting that IBCs in the human bladder may be associated with recurrent UTI and possibly with chronic LUTS, it is certainly possible that IBCs may not affect these disease processes or have only a limited role. In a study using fluorescence in situ hybridization to evaluate bladder biopsy specimens of patients with neurogenic bladder and a history of chronic recurrent bacteruria, no evidence of bacterial reservoirs was identified. ${ }^{46}$ However, these findings may be due to limited sampling with only a small tissue specimen or a result of the reservoirs remaining metabolically inactive and therefore not easily detected with RNA probes.

If future research provides adequate evidence that IBCs do have a role in recurrent UTIs and chronic LUTS in humans, current diagnostic and treatment approaches will need to be reexamined. Longer courses of antibiotics with good intracellular penetration may be used to rule out occult bacterial infection before a diagnosis of OAB or IC/BPS is assigned to a patient with persistent LUTS. Although prolonged courses of antibiotics may be costly, increase the risk of development of resistant organisms, and have potential adverse effects, a course of up to several months might not be unreasonable considering the very slow turnover of urothelial cells ( $>200$ days). This duration of therapy may be required to completely eradicate bacteria occupying intracellular reservoirs. ${ }^{47}$ 
Extended therapy may also be considered for patients with persistently positive urine cultures who have no other structural or reversible source for recurrent UTIs. In the mouse model, a 10-day course of trimethoprimsulfamethoxazole therapy was more effective at reducing the recurrence of UTI than a 3-day course, although neither course completely eradicated UPEC from the bladder. ${ }^{6}$ Another study of 17 antibiotics from 7 different functional classes found that most antibiotics were at least moderately effective against bacteria in in vivo biofilms, but only a few antibiotics, such as fluoroquinolones and nitrofurantoin, were able to eliminate intracellular bacteria in tissue cultureebased assays. ${ }^{48}$ However, in mice with appropriate levels of antibiotics in the urine, UPEC reservoirs were not completely eliminated by any of the antibiotics tested.

Ultimately, investigation of new approaches for targeting stationary-phase bacteria in quiescent reservoirs, capable of eradication of intracellular biofilms, will be necessary. Integration of knowledge on IBC formation in the urinary tract into our approach to patients with persistent LUTS and recurrent UTIs might lead to a much anticipated paradigm shift and thus more successful diagnostic and treatment strategies for these patients.

\section{References}

1. Coyne KS, Sexton CC, Thompson CL, et al. The prevalence of lower urinary tract symptoms (LUTS) in the USA, the UK and Sweden: results from the Epidemiology of LUTS (EpiLUTS) study. BJU Int. 2009; 104:352-360. [PubMed: 19281467]

2. Abrams P, Cardozo L, Fall M, et al. The standardisation of terminology of lower urinary tract function: report from the Standardisation Sub-committee of the International Continence Society. Neurourol Urodyn. 2002; 21:167-178. [PubMed: 11857671]

3. Anderson GG, Palermo JJ, Schilling JD, et al. Intracellular bacterial biofilm-like pods in urinary tract infections. Science. 2003; 301:105-107. [PubMed: 12843396]

4. Justice SS, Hung C, Theriot JA, et al. Differentiation and developmental pathways of uropathogenic Escherichia coli in urinary tract pathogenesis. Proc Natl Acad Sci U S A. 2004; 101:1333-1338. [PubMed: 14739341]

5. Mulvey MA, Schilling JD, Martinez JJ, Hultgren SJ. Bad bugs and beleaguered bladders: interplay between uropathogenic Escherichia coli and innate host defenses. Proc Natl Acad Sci U S A. 2000; 97:8829-8835. [PubMed: 10922042]

6. Schilling JD, Lorenz RG, Hultgren SJ. Effect of trimethoprimsulfamethoxazole on recurrent bacteriuria and bacterial persistence in mice infected with uropathogenic Escherichia coli. Infect Immun. 2002; 70:7042-7049. [PubMed: 12438384]

7. de Groat W. A neurologic basis for the overactive bladder. Urology. 1997; 50(suppl 6A):36-52. [PubMed: 9426749]

8. Brading AF. A myogenic basis for the overactive bladder. Urology. 1997; 50(suppl 6A):57-67. [PubMed: 9426752]

9. Berry SH, Elliott MN, Suttorp M, et al. Prevalence of symptoms of bladder pain syndrome/ interstitial cystitis among adult females in the United States. J Urol. 2011; 186:540-544. [PubMed: 21683389]

10. Elbadawi A. Interstitial cystitis: a critique of current concepts with a new proposal for pathologic diagnosis and pathogenesis. Urology. 1997; 49(5A suppl):14-40. [PubMed: 9145999]

11. Duncan JL, Schaeffer AJ. Do infectious agents cause interstitial cystitis? Urology. 1997; 49(5A suppl):48-51. [PubMed: 9146001]

12. Warren JW, Horne L, Hebel JR, et al. Pilot study of sequential oral antibiotics for the treatment of interstitial cystitis. J Urol. 2000; 163:1685-1688. [PubMed: 10799160]

13. Khasriya R, Bignall J, Khan S, Malone-Lee J. Routine MSU culture in patients with symptoms of OAB may be missing many genuine infections. Int Urogynecol J. 2008; 19:S48-S49. 
14. Drekonja DM, Johnson JR. Urinary tract infections. Prim Care. 2008; 35:345-367. vii. [PubMed: 18486719]

15. Durier, JL. Presented at the Women's Urological Health Program, National Institute of Diabetes, Digestive and Kidney Diseases Research Symposium on Interstitial Cystitis. Bethesda, Maryland: Jan 9-11. 19951995 Anti-anaerobic antibiotic use in chronic inflammation, urgency-frequency, urge incontinence and in interstitial cystitis syndromes.

16. Warren JW, Horne LM, Hebel R, et al. Pilot study of sequential oral antibiotics for the treatment of interstitial cystitis. J Urol. 2000; 163:1636-1688.

17. Burkhard FC, Blick N, Hochreiter WW, Studer UE. Urinary urgency and frequency, and chronic urethral and/or pelvic pain in females. Can doxycycline help? J Urol. 2004; 172:232-235. [PubMed: 15201781]

18. Potts JM, Ward AM, Rackley RR. Association of chronic urinary symptoms in women and Ureaplasma urealyticum. Urology. 2000; 55:486-489. [PubMed: 10736488]

19. Kass EH. Asymptomatic infections of the urinary tract. 1956. J Urol. 2002; 167:1019-1021.

20. Stamm WE, Counts GW, Running KR, et al. Diagnosis of coliform infection in acutely dysuric women. N Engl J Med. 1982; 307:463-468. [PubMed: 7099208]

21. Fihn S. Acute uncomplicated urinary tract infection in women. N Engl J Med. 2003; 349:259-266. [PubMed: 12867610]

22. Grabe M, Bjerklund-Johansen T, Botto H, et al. Guidelines on Urologic Infections. Available at: http://www.uroweb.org, 2009; Accessed July 10, 2014.

23. Kodner CM, Gupton EK. Recurrent urinary tract infections in women: diagnosis and management. Am Fam Physician. 2010; 82:638-643. [PubMed: 20842992]

24. Kunin CM, White LV, Hua TH. A reassessment of the importance of "low-count" bacteriuria in young women with acute urinary symptoms. Ann Intern Med. 1993; 119:454-460. [PubMed: 8357110]

25. Hooton TM, Roberts PL, Cox ME, Stapleton AE. Voided midstream urine culture and acute cystitis in premenopausal women. N Engl J Med. 2013; 369:1883-1891. [PubMed: 24224622]

26. Hilt EE, McKinley K, Pearce MM, et al. Urine is not sterile: use of enhanced urine culture techniques to detect resident bacterial flora in the adult female bladder. J Clin Microbiol. 2014; 52:871-876. [PubMed: 24371246]

27. Pearce MM, Hilt EE, Rosenfeld AB, et al. The female urinary microbiome: a comparison of women with and without urgency urinary incontinence. MBio. 2014; 5:e1283-e1214.

28. Wolfe AJ, Toh E, Shibata N, et al. Evidence of uncultivated bacteria in the adult female bladder. J Clin Microbiol. 2012; 50:1376-1383. [PubMed: 22278835]

29. Hvidberg H, Struve C, Krogfelt KA, et al. Development of a longterm ascending urinary tract infection mouse model for antibiotic treatment studies. Antimicrob Agents Chemother. 2000; 44:156-163. [PubMed: 10602738]

30. Anderson GG, Dodson KW, Hooton TM, Hultgren SJ. Intracellular bacterial communities of uropathogenic Escherichia coli in urinary tract pathogenesis. Trends Microbiol. 2004; 12:424-430. [PubMed: 15337164]

31. Anderson GG, Goller CC, Justice S, et al. Polysaccharide capsule and sialic acid-mediated regulation promote biofilm-like intracellular bacterial communities during cystitis. Infect Immun. 2010; 78:963-975. [PubMed: 20086090]

32. Rosen DA, Pinkner JS, Jones JM, et al. Utilization of an intracellular bacterial community pathway in Klebsiella pneumoniae urinary tract infection and the effects of FimK on type 1 pilus expression. Infect Immun. 2008; 76:3337-3345. [PubMed: 18411285]

33. Barber AE, Norton JP, Spivak AM, Mulvey MA. Urinary tract infections: current and emerging management strategies. Clin Infect Dis. 2013; 57:719-724. [PubMed: 23645845]

34. Mulvey MA, Schilling JD, Hultgren SJ. Establishment of a persistent Escherichia coli reservoir during the acute phase of a bladder infection. Infect Immun. 2001; 69:4572-4579. [PubMed: 11402001]

35. Cooper EH. The biology of bladder cancer. Ann R Coll Surg Engl. 1972; 51:1-16. [PubMed: 5043669] 
36. Garofalo CK, Hooton TM, Martin SM, et al. Escherichia coli from urine of female patients with urinary tract infections is competent for intracellular bacterial community formation. Infect Immun. 2007; 75:52-60. [PubMed: 17074856]

37. Rosen DA, Hooton TM, Stamm WE, et al. Detection of intracellular bacterial communities in human urinary tract infection. PLoS Med. 2007; 4:e329. [PubMed: 18092884]

38. Robino L, Scavone P, Araujo L, et al. Intracellular bacteria in the pathogenesis of Escherichia coli urinary tract infection in children. Clin Infect Dis. 2014; 59:e158-e164. [PubMed: 25091303]

39. Kelley SP, Courtneidge HR, Birch RE, et al. Urinary ATP and visualization of intracellular bacteria: a superior diagnostic marker for recurrent UTI in renal transplant recipients? Springerplus. 2014; 3:200. [PubMed: 24839587]

40. Robino L, Scavone P, Araujo L, et al. Detection of intracellular bacterial communities in a child with Escherichia coli recurrent urinary tract infections. Pathog Dis. 2013; 68:78-81. [PubMed: 23733378]

41. Horsley H, Malone-Lee J, Holland D, et al. Enterococcus faecalis subverts and invades the host urothelium in patients with chronic urinary tract infection. PloS One. 2013; 8:e83637. [PubMed: 24363814]

42. Russo TA, Stapleton AE, Wenderoth S, et al. Chromosomal restriction fragment length polymorphism analysis of Escherichia coli strains causing recurrent urinary tract infections in women. J Infect Dis. 1995; 172:440-445. [PubMed: 7622887]

43. Ikaheimo R, Siitonen A, Heiskanen T, et al. Recurrence of urinary tract infection in a primary care setting: analysis of a 1-year followup of 179 women. Clin Infect Dis. 1996; 22:91-99. [PubMed: 8824972]

44. Brauner A, Jacobson SH, Kuhn I. Urinary Escherichia causing recurrent infectionsea prospective follow-up of biochemical phenotypes. Clin Nephrol. 1992; 38:318-323. [PubMed: 1468162]

45. Cass AS, Ireland GW. Antibacterial perineal washing for prevention of recurrent urinary tract infections. Urology. 1985; 25:492-494. [PubMed: 3992773]

46. Schlager TA, Hendley JO, Peters CA. Absence of bacterial reservoirs in the bladder epithelium of patients with chronic bacteriuria due to neurogenic bladder. J Urol. 2009; 182(4 Suppl):17141719. [PubMed: 19692065]

47. Blango MG, Mulvey MA. Persistence of uropathogenic Escherichia coli in the face of multiple antibiotics. Antimicrob Agents Chemother. 2010; 54:1855-1863. [PubMed: 20231390]

48. Cooper EH. The biology of bladder cancer. Ann R Coll Surg Engl. 1972; 51:1-16. [PubMed: 5043669] 


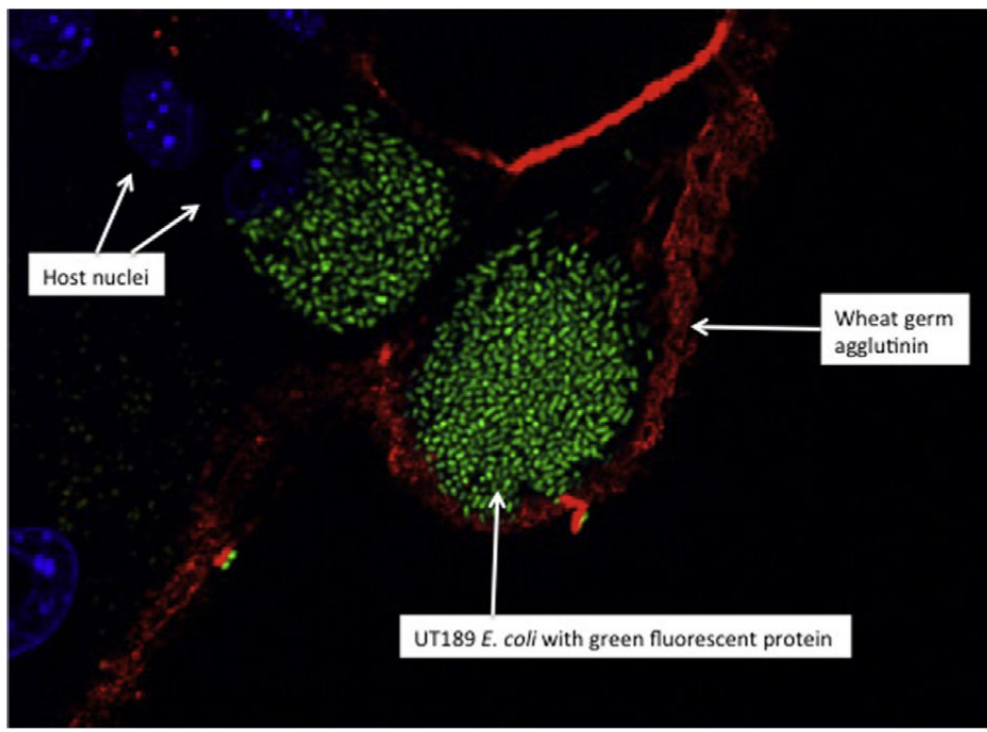

Figure 1.

Fluorescence micrograph of uropathogenic Escherichia coli (green) growing as an intracellular bacterial community during experimental cystitis. A female mouse was transurethrally infected with UTI89 carrying a plasmid-encoded green fluorescent protein (green). The bladder was harvested, bisected, and fixed in 3\% paraformaldehyde. The surface was visualized with wheat germ agglutinin (red), and the host nucleus was visualized with Hoechst (blue). (Color version available online). 


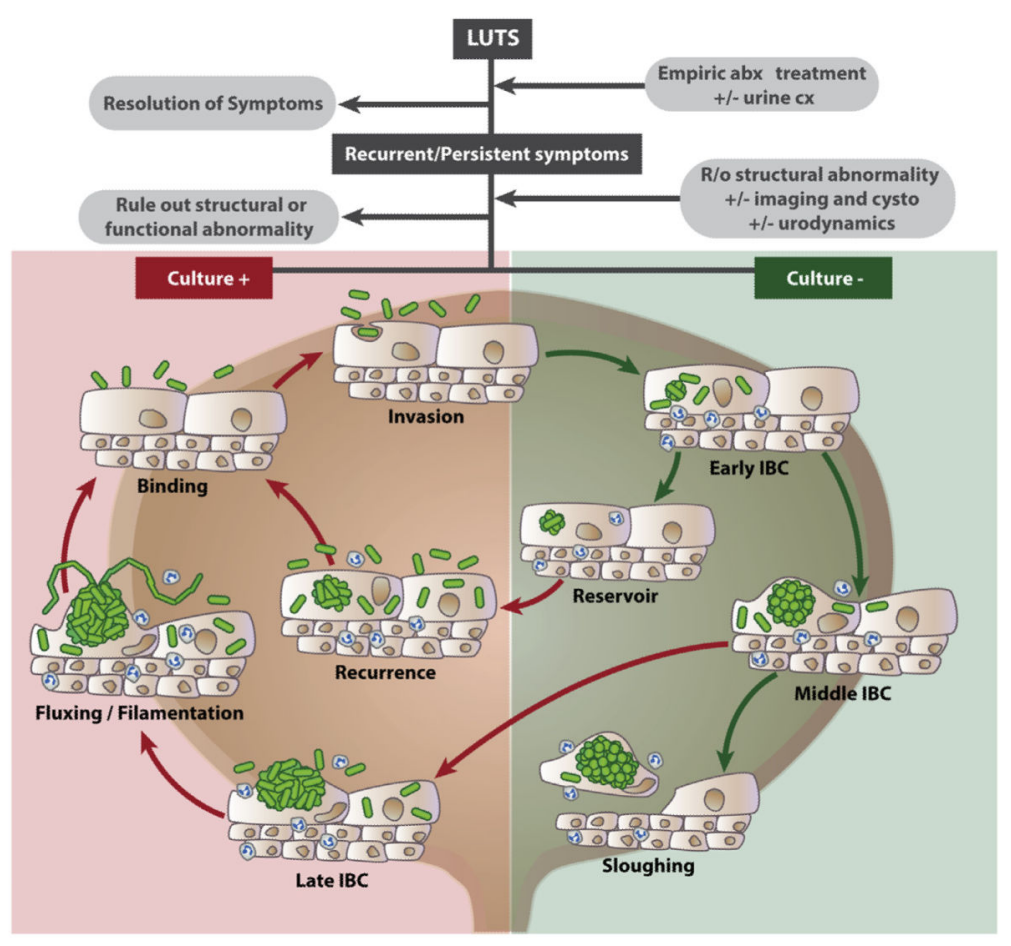

Figure 2.

Diagram including flow chart of clinical management of patients with lower urinary tract symptoms (LUTS) and corresponding cycle of intracellular bacterial community (IBC) maturation and development. Patients with an initial episode of LUTS will likely be diagnosed with a urinary tract infection by a primary care physician and treated with a course of antibiotics. If symptoms persist or recur and bother the patient, she will likely be referred to a urologist. This evaluation may include a combination of imaging, cystoscopy, or urodynamics, if indicated. If no structural or functional sources for symptoms are identified, the patient's diagnosis is ultimately determined by the urine culture. The red half of the bladder (left half under "Culture +") shows phases of IBC development, during which bacteria are in the intraluminal state in the bladder and a patient would be likely to have a positive urine culture with bacteria susceptible to antibiotics. The green half of the bladder (right half under "Culture -") shows phases of IBC development during which bacteria are intracellular and a patient would be likely to have a negative urine culture with bacteria protected from antibiotic therapy. (Color version available online). 